\title{
An Enhanced Power Based MAC Protocol for Wireless Networks
}

\author{
S.L. Prathapa Reddy ${ }^{1}$, M.V. Subramanyam ${ }^{2}$, K. Satyaprasad ${ }^{3}$ \\ ${ }^{I}$ Assistant professor, Dept. of ECE, K.O.R.M.C.E, Kadapa- 516003, AP, India, \\ ${ }^{2}$ Principal, Dept. of ECE, Santhiram Engineering College, Nandyal-518501, A.P, India. \\ ${ }^{3}$ Professor, Dept. of ECE, J.N.T. University Kakinada, Kakinada-533003, A.P, India
}

\begin{abstract}
Power based connectivity is an ad hoc research topic. Implementing power control mechanisms enhance network life and to improve throughput, locate cost effective routes and spatial reuse. This paper uses MAC protocol to implement coordination functions and power control mechanisms through the use of fuzzy rules generated by upper network layers using two input variables like link quality and node neighborhood count with optimal power consumption level as the output variable. Experiments using fuzzy rules are compared to DSR routing and two hop power control methods. Result showed that two hop power control with fuzzy logic method yields lower route discovery time, increased cache replies, minimum simulation time and end to end delay when compared to DSR routing and two hop routing protocol.

Keywords: Power Control, Medium Access Control (MAC), Energy Saving, Fuzzy logic
\end{abstract}

\section{Introduction}

An ad hoc network consists of small, mobile devices using wireless medium to communicate amongst themselves. Most ad hoc networks have no fixed infrastructure or centralized administration. As nodes join/leave or roam the network, network topology is dynamic. The network self organizes and self configures and so differs from wired networks. Ad hoc network applications range from disaster recovery, distributed computing, and battlefield surveillance. Most nodes have limited battery power and bandwidth. Routing protocols are not required [1] when 2 nodes are within transmission range of each other.

Some ad hoc network advantages are the following:

- No central network administration,

- Self-configuring nature (nodes act as routers),

- Self-healing through periodic re-configuration,

- Scalable when nodes are added,

- Flexibility (being accessible using Internet from varied, multiple locations).

\section{Its limitations include:}

- Every node should cooperate to ensure a high performance,

- Throughput depends on system load,

- Reliability maintenance requires enough available nodes,

- Huge latency/time delay is experienced in large networks [2]

As wireless medium is used by nodes, it results in collision without successful transmission when multiple hosts attempt to forward data simultaneously. Hence, recourse is taken to medium access control (MAC) protocol, to control access to shared wireless medium, avoiding collisions, and maintaining coordinated medium access [3].

MAC layer performs flow control, error detection, framing, correction, and physical addressing. This layer includes functions/procedures required for transmitting data between two or more network nodes. MAC protocol design should tackle issues generated by node roaming and unreliable time varying channels [4].

Carrier Sense Multiple Access (CSMA) avoids collisions. When a node wants to send a packet it listens to medium, for a fixed time. When no traffic is sensed, it then starts to transmit. When 2 two nodes transmit simultaneously without knowledge about other's transmission, it leads to a collision. But CSMA-style MAC protocols ensure channel utilization of around 50\% - 80\% based on access policy used [5].

As ad hoc network nodes are battery operated devices, energy conservation, and power control are the main aims. Ad hoc networks power consumption is reduced by either controlling transmission power or choosing optimal transmission [6] routes. Power based connectivity is a recent concept in wireless ad-hoc networks. Reducing the power consumption at each node will increase the end-to-end throughput. A node's power availability controls transmission power which in turn affects signal quality and determines neighboring nodes status. Hence, this affects network layer, as interference leads to congestion affecting transport layer. 
MAC protocols are designed to decide maximum transmission power to forward request-to-send (RTS), and clear-to-send (CTS) packets to save energy thereby determining minimum power needed for data transmission and packet acknowledgement (ACK). Power required for communication is specified through three components: They are $\mathrm{P}_{\text {Rxelec }}, \mathrm{P}_{\text {Txelec }}$, and $\mathrm{P}_{\text {TxRad }}(\mathrm{p})$. $\mathrm{P}_{\text {Rxelec }}$ is power consumed by node receiver, $\mathrm{P}_{\text {Txelec }}$ is power consumed by transmitter electronics, and $\mathrm{P}_{\text {TxRad }}(\mathrm{p})$ is power consumed by power amplifier for packet transmission at power level, where $\mathrm{p}$ is the actual power radiated [7].

Energy consumption when packet is transmitted through the use of hops from sender node to receiver node with distance $\mathrm{d}$, is given by

$$
(d / r)\left(P_{\text {Rxelec }}+P_{\text {Txelec }}+c r^{\alpha}\right)
$$

which is minimized at,

$$
r_{\text {crit }}=\sqrt[\alpha]{\frac{P_{\text {Rxelec }}+P_{\text {Txelec }}}{c(\alpha-1)}}
$$

To satisfy network connectivity, available power range should be greater than $r_{\text {crit }}[8,9]$.

Power Saving processes proposes the use of 2 varying power states at a node [10]:

1) Awake: wireless interface of a node is powered to transmit/receive. In this state, a node transmits/receives or is idle.

2) Doze: The node's wireless interface is powered down when it can neither transmit/receive.

\section{Related Work}

Energy Efficient Routing Protocol with Adaptive Fuzzy Threshold Energy for MANETs [11] was presented by Hiremath and Joshi. MANET's life was affected by node life. A new on-demand routing based protocol was proposed to conserve energy in mobile nodes, to increase MANET life, the suggested methodology being based on adaptive fuzzy thresholding of residual nodes energy, participating in route discovery from sender to receiver. Experiments were undertaken, and results compared to Load-Aware Energy Efficient Protocol (LAEE) protocol which proved that AFTE was better than LAEE. Improvement in average network life was $13 \%$ at the first node failure, $15 \%$ when $50 \%$ node failure was considered and $23 \%$ when $100 \%$ node failure was considered in comparison to LAEE.

Fuzzy-controlled Power-aware Routing Protocol (FPRP) for Mobile Ad Hoc Networks [12] was presented by Banerjee and Dutta. Routing decisions dynamically at nodes to form a closer-optimal powerefficient end-to end route to forward data packets in Fuzzy-controlled power aware routing protocol (FPRP). The protocol was distributed so that only neighboring nodes location information was exploited in every routing node. Routers life status was measured through a fuzzy controller called route decider. Fuzzy controller used rate of depletion, residual charge, communication load, and node proximity. Simulation showed FPRP produced major improvements when compared with other power aware ad hoc network routing protocols, even when node numbers exceeded 2000.

A reliable Energy-Efficient Multi-Level Routing Algorithm for Sensor Networks [13] was presented by $\mathrm{Yu}$, Fuzzy Petri nets selected cluster heads in this knowledge-based inference approach. Fuzzy logic's reasoning method calculated reliability degree in route budding tree from cluster heads to the base station. Hence, the best and reliable route among cluster heads was constructed. The algorithm provided an idea to balance each node's energy load, providing global reliability for the network. Every iteration has three phases: clustering phase, multi-hop routing phase and data transmission phase. In clustering phase, a cluster heads set was elected, and remaining nodes were cluster members. In the second phase, multi-hop route was generated while in the data transmission phase, every cluster member node forwarded a specific data amount to the cluster head. All cluster heads aggregated received data and forwarded them to the base station through multi-hop routing. Simulation results demonstrated that network life was prolonged, and energy consumption reduced.

An Adaptive Power Control Based Spectrum Handover for Cognitive Radio Networks [14] was presented by $\mathrm{Lu}$, et al., which proposed a spectrum handover scheme combining dynamic spectrum allocation and power control to reduce unnecessary handovers. This procedure enhanced overall network performance, improving spectral efficiency. Users were split into primary users (PU) and secondary users (SUs). SUs used licensed channels till primary users (PUs) aggregate interference, did not exceed predetermined thresholds. When a PU arrived, an SU calculated the maximum transmission power that the SU did not interfere with the $\mathrm{PU}$, if the SU could reach its receiver and continue transmission with reduced power. Otherwise, it switched over to an idle band. Experiments showed this scheme reduced spectrum handover ratio and improved effective data rate by $30 \%$. 
A Fuzzy Logic Approach to Beaconing for Vehicular Ad hoc Networks [15] was presented by Ghafoor, et al. Vehicular Ad Hoc Network (VANET) is a new technology used for intra-vehicular communication when fixed infrastructures were absent. An Adaptive Beaconing Rate (ABR) approach was for VANETs based on fuzzy logic to control beaconing frequency by considering current traffic characteristics. ABR took one direction vehicles percentage and their status as fuzzy decision making system inputs. Beaconing rate tuning is based on vehicular traffic characteristics.

\section{Materials And Methods}

Fuzzy logic (FL) is a reasoning approach, specifying degrees of truthiness instead of Boolean value (true or false) used by computers. Fuzzy logic has 0 and 1 as extreme cases of true and false respectively and also includes truth's various states in between. FL nearly resembles human thinking.

FL is implemented as follows [16]:

1. Fuzzification - This transforms crisp data into fuzzy data/Membership Functions.

2. Fuzzy Inference Process - This combines membership functions with if-then rules to reach a fuzzy output.

3. Defuzzification - This uses various methods to calculate different outputs storing them in a lookup table. When an application in executed, output is taken from the lookup table based on the current input variables

A fuzzy [17] IF-THEN rule includes an IF part (antecedent) and THEN part (consequent) where antecedent combines two or more terms, and consequent one term.

Fuzzy logic/fuzzy sets are techniques to control uncertainty in many applications. Fuzzy logic is used by MAC layer to control nodes power consumption in this work. A network layers upper layer generates fuzzy rules. Neighbourhood node count and link quality parameters are inputs in fuzzy rule formation. The neighbourhood node count's numeric values are represented in 3 terms, low, medium and high. The same 3 terms also represent link quality. Power level to be used by a node is specified by states of very low, low, medium, high and very high link quality. A node's power usage is based on link quality and neighbourhood nodes number.

Block diagram for the work is given:

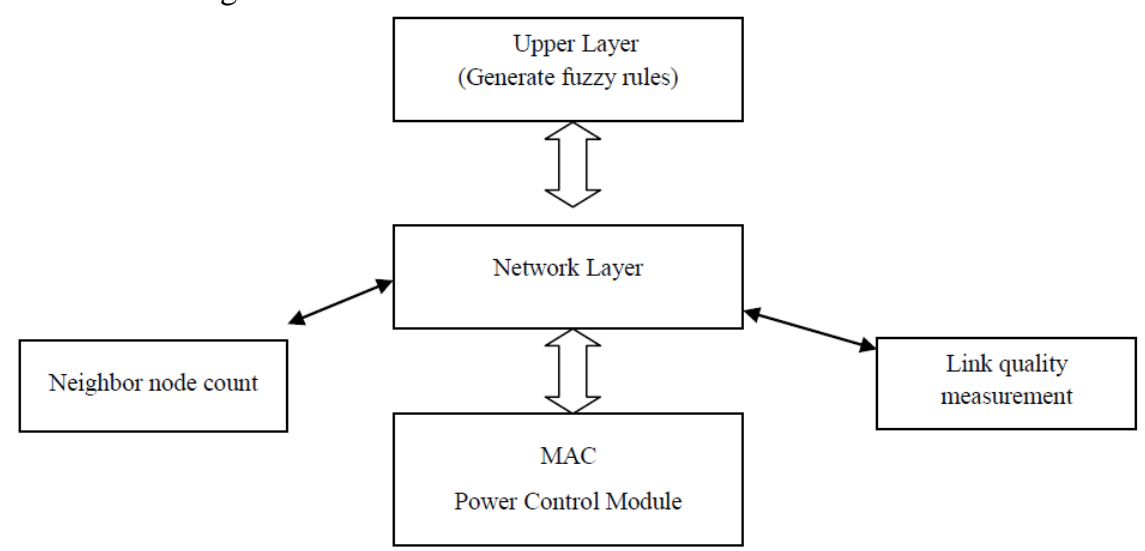

Figure 1: Block diagram of proposed approach

\section{Experimental Setup And Results}

For the experiments link quality and neighbor node count are taken from the definition points, and shape parameters given below and termed as three states low, medium and high. Output variable power is taken from the definition points and termed as states very low, low, medium, high and very high.

\subsection{Input Variable "LQ"}

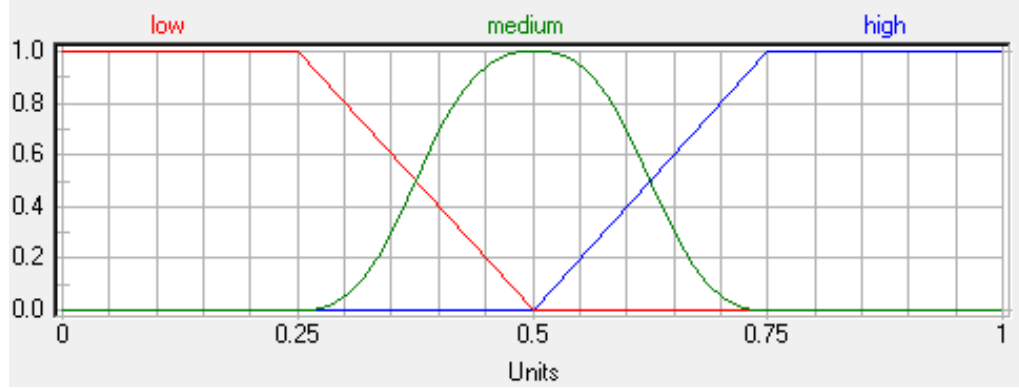

Figure 1: MBF of "LQ" 
Table 1: Definition Points of MBF "LQ

\begin{tabular}{|l|l|l|l|l|}
\hline Term Name & Shape/Par. & \multicolumn{4}{|l|}{ Definition Points (x, y) } \\
\hline Low & Linear & $(0,1)$ & $(0.25,1)$ & $(0.5,0)$ \\
\hline & & $(1,0)$ & & \\
\hline medium & S-Shape/0.50 & $(0,0)$ & $(0.25,0)$ & $(0.5,1)$ \\
\hline & & $(0.75,0)$ & $(1,0)$ & \\
\hline High & Linear & $(0,0)$ & $(0.5,0)$ & $(0.75,1)$ \\
\hline & & $(1,1)$ & & \\
\hline
\end{tabular}

\subsection{Input Variable "NNC"}

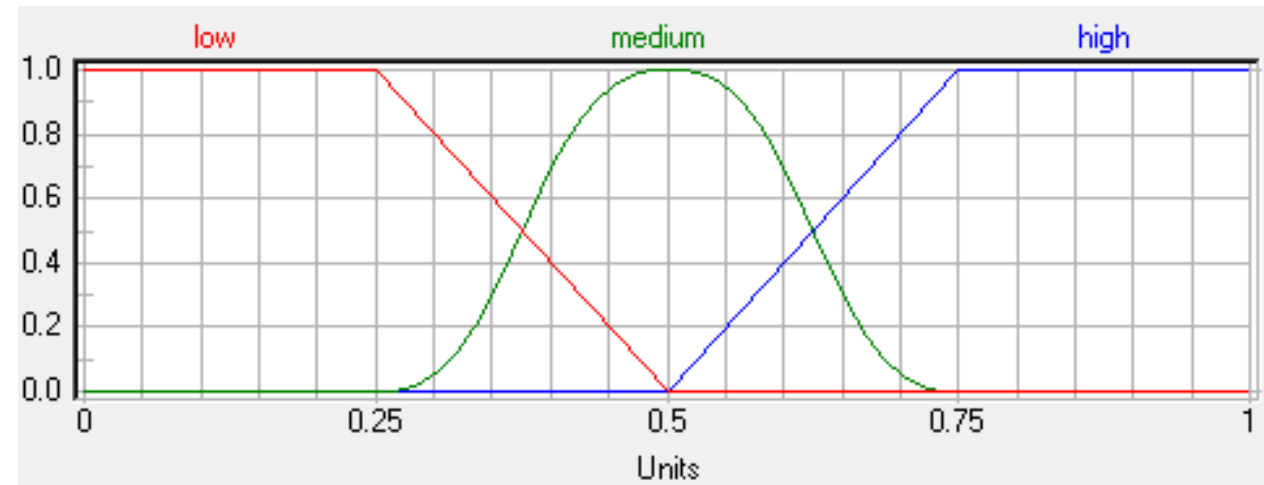

Figure 2: MBF of "NNC"

Table 2: Definition Points of MBF "NNC"

\begin{tabular}{|l|l|l|l|l|}
\hline Term Name & Shape/Par. & \multicolumn{4}{|l|}{ Definition Points (x, y) } \\
\hline Low & Linear & $(0,1)$ & $(0.25,1)$ & $(0.5,0)$ \\
& & $(1,0)$ & & \\
\hline medium & S-Shape/0.50 & $(0,0)$ & $(0.25,0)$ & $(0.5,1)$ \\
& & $(0.75,0)$ & $(1,0)$ & \\
\hline High & Linear & $(0,0)$ & $(0.5,0)$ & $(0.75,1)$ \\
& & $(1,1)$ & & \\
\hline
\end{tabular}

\subsection{Output Variable "Power"}

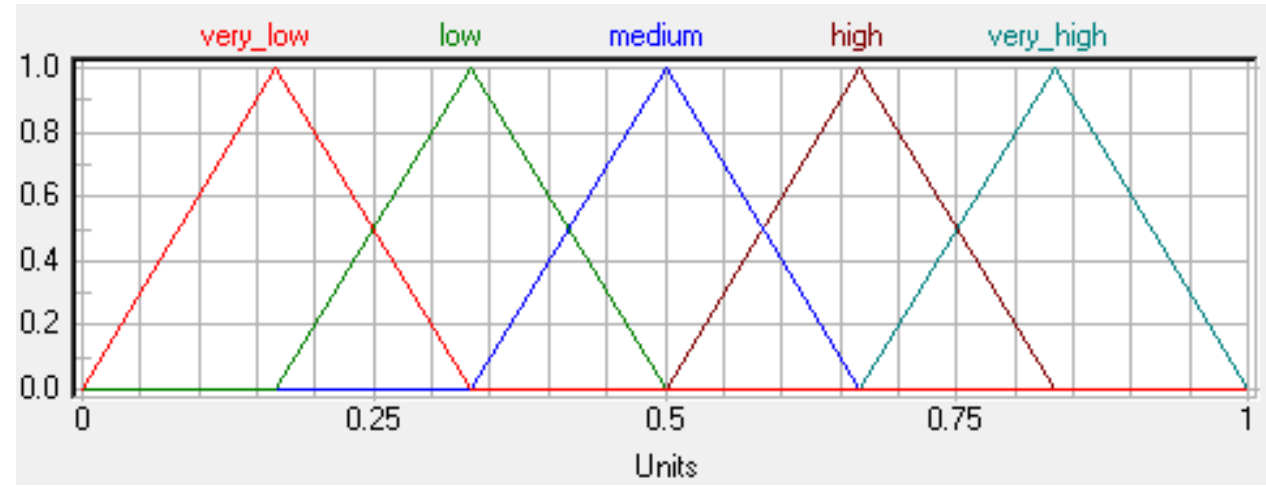

Figure 3: MBF of "Power"

Table 3: Definition Points of MBF "Power"

\begin{tabular}{|l|l|l|l|l|}
\hline Term Name & Shape/Par. & \multicolumn{2}{|l|}{ Definition Points $(\mathbf{x}, \mathbf{y})$} & $(0.16666,1)$ \\
\hline very_low & linear & $\begin{array}{l}(0,0) \\
(1,0)\end{array}$ & $(0.33334,0)$ \\
\hline Low & linear & $(0,0)$ & $\begin{array}{l}(0.16666,0) \\
(1,0)\end{array}$ & $(0.33334,1)$ \\
& & $(0.5,0)$ & $(0.33334,0)$ & $(0.5,1)$ \\
\hline medium & linear & $(0,0)$ & $(1,0)$ & \\
\hline High & & $(0.66666,0)$ & $(0.5,0)$ & $(1,0)$ \\
& linear & $(0,0)$ & $(0.666666,0)$ & $(0.83334,1)$ \\
\hline very_high & linear & $(0.83334,0)$ & & \\
& & $(1,0)$ & & \\
\hline
\end{tabular}

\subsection{Rule Block "RB1"}

Using various combinations of link quality and neighborhood node count levels, minmax aggregation is used to form a set of rules. 


\section{Parameter}

\begin{tabular}{|l|l|}
\hline Aggregation: & MINMAX \\
\hline Parameter: & 0.00 \\
\hline Result Aggregation: & MAX \\
\hline Number of Inputs: & 2 \\
\hline Number of Outputs: & 1 \\
\hline Number of Rules: & 45 \\
\hline
\end{tabular}

Table 4: Rules of the Rule Block "RB1"

\begin{tabular}{|c|c|c|c|}
\hline \multicolumn{2}{|l|}{ IF } & \multicolumn{2}{|c|}{ THEN } \\
\hline LQ & NNC & DoS & Power \\
\hline Low & low & 0.20 & very_low \\
\hline Low & low & 0.63 & low \\
\hline Low & low & 0.14 & medium \\
\hline Low & low & 0.74 & high \\
\hline Low & low & 0.27 & very_high \\
\hline Low & medium & 0.13 & very_low \\
\hline Low & medium & 0.51 & low \\
\hline Low & medium & 0.16 & medium \\
\hline Low & medium & 0.23 & high \\
\hline Low & medium & 0.12 & very_high \\
\hline Low & high & 0.09 & very_low \\
\hline Low & high & 0.96 & low \\
\hline Low & high & 0.48 & medium \\
\hline Low & high & 0.38 & high \\
\hline Low & high & 0.29 & very_high \\
\hline Medium & low & 0.27 & very_low \\
\hline Medium & low & 0.30 & low \\
\hline Medium & low & 0.60 & medium \\
\hline Medium & low & 0.91 & high \\
\hline Medium & low & 0.55 & very_high \\
\hline Medium & medium & 0.84 & very_low \\
\hline Medium & medium & 0.13 & low \\
\hline Medium & medium & 0.97 & medium \\
\hline Medium & medium & 0.17 & high \\
\hline Medium & medium & 0.60 & very_high \\
\hline Medium & high & 0.20 & very_low \\
\hline Medium & high & 0.18 & low \\
\hline Medium & high & 0.14 & medium \\
\hline Medium & high & 0.34 & high \\
\hline Medium & high & 0.68 & very_high \\
\hline High & low & 0.80 & very_low \\
\hline High & low & 0.52 & low \\
\hline High & low & 0.92 & medium \\
\hline High & low & 0.34 & high \\
\hline High & low & 0.09 & very_high \\
\hline High & medium & 0.62 & very_low \\
\hline High & medium & 0.86 & Low \\
\hline High & medium & 0.71 & Medium \\
\hline High & medium & 0.61 & High \\
\hline High & medium & 0.47 & very_high \\
\hline High & high & 0.09 & very_low \\
\hline High & high & 0.51 & Low \\
\hline High & high & 0.30 & Medium \\
\hline High & high & 0.59 & High \\
\hline High & high & 0.73 & very_high \\
\hline
\end{tabular}

For comparing, the performance of the proposed fuzzy logic two hop power conserving method, average route discovery time, end to end delay and simulation time are used as parameters. Results are shown from figure 5 to figure 8. Result reveals that proposed fuzzy logic method yields less route discovery time, increased cache replies, minimum simulation time and end to end delay when comparing to DSR routing and two hop routing protocol.

Proposed fuzzy route discovery time is $0.55 \mathrm{sec}$ and is drastically less when compared to other method. 


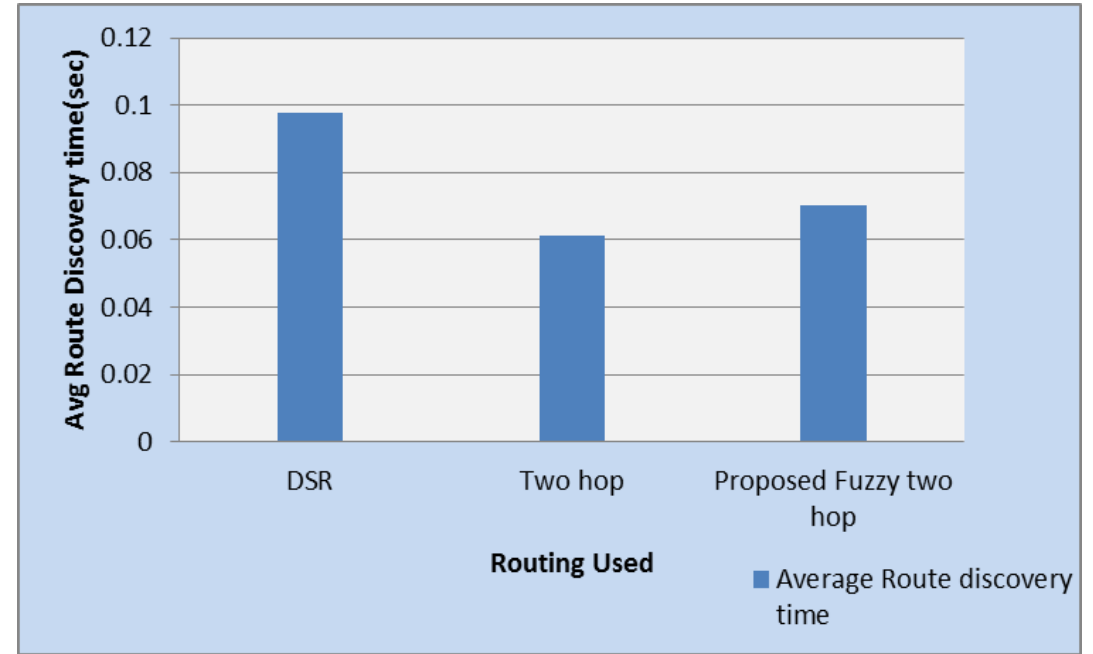

Figure 5 : Average Route discovery time

Proposed fuzzy method provides increase in average cached replies

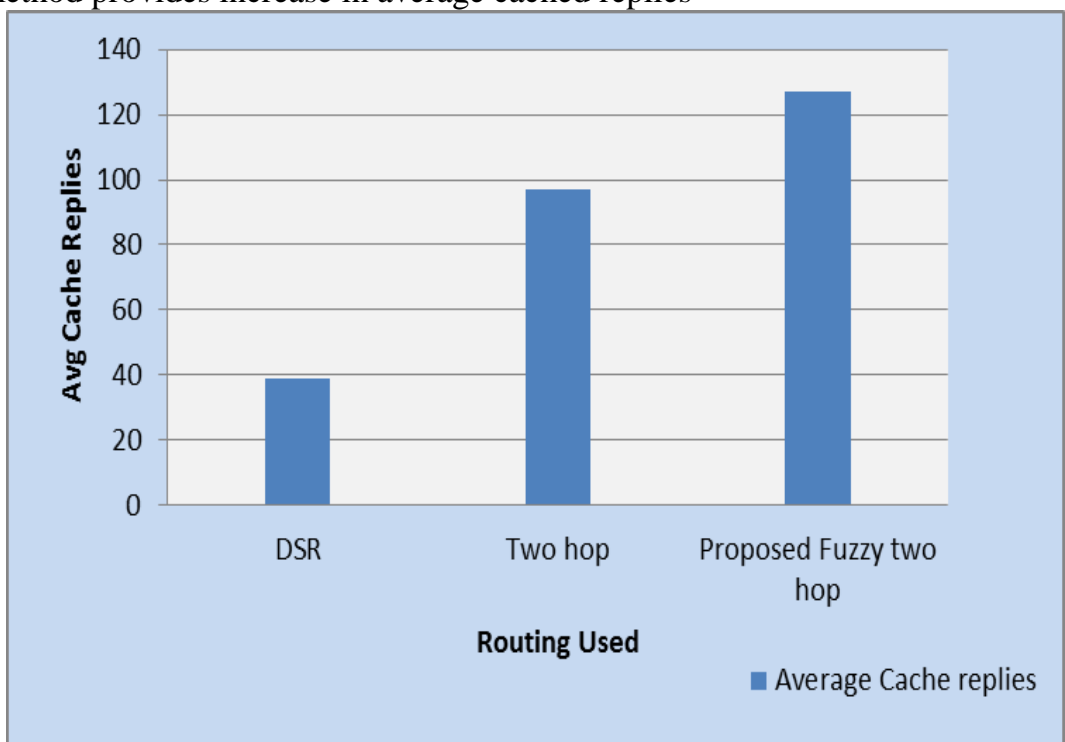

Figure 6 : Average cache replies

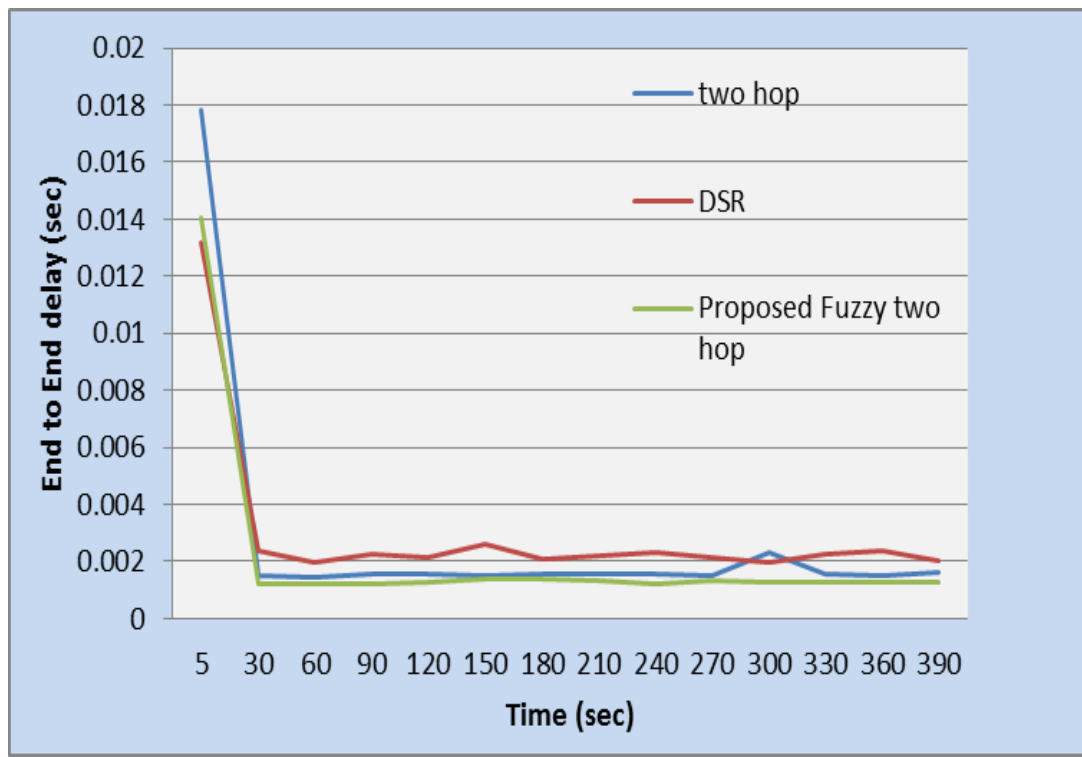

Figure 7 : End to end delay Vs Simulation time 


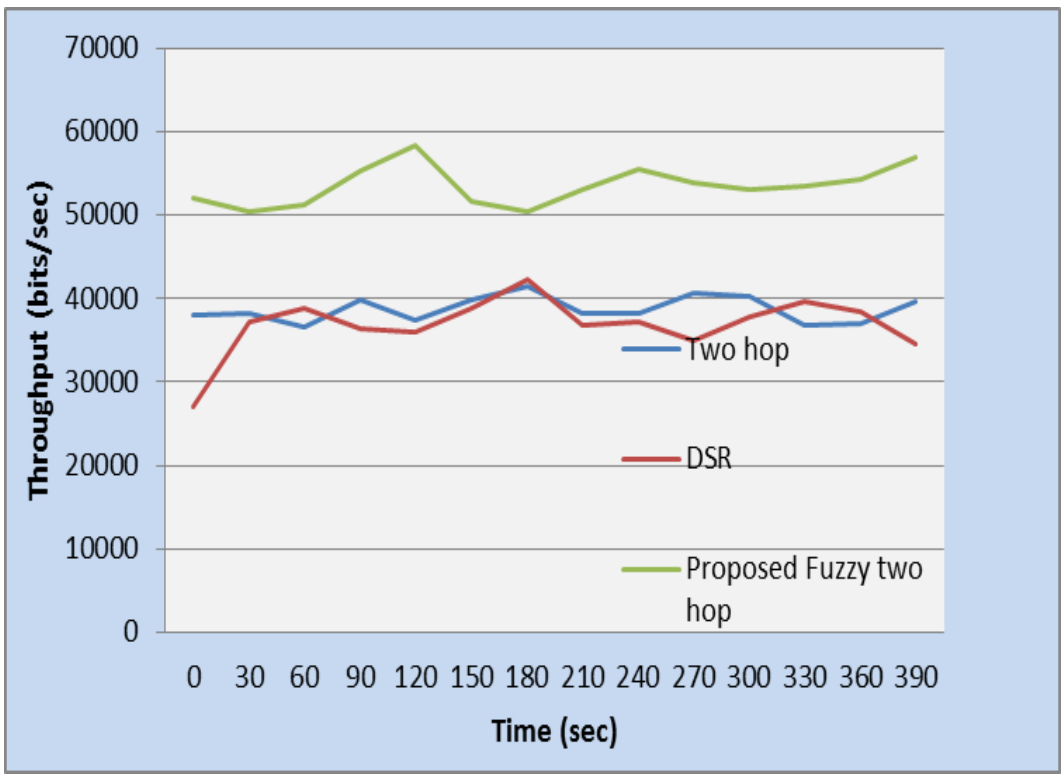

Figure 8: Throughput Vs simulation time'

\section{Conclusion}

Every ad hoc network node is a battery powered device and so energy conservation and power reduction are major goals. Ad hoc networks power consumption is controlled by controlling transmission power or choosing optimal transmission routes. Fuzzy logic is similar to human reasoning when some things are uncertain. Fuzzy Logic derives power control at each network node through fuzzy rules generated by input variables link quality and neighborhood count in this work. Experiments using fuzzy rules are compared to DSR routing and simple 2 hop power control procedures. The results revealed that 2 hop power controls with fuzzy logic procedures used reduced route discovery time, increased cache replies with limited simulation time and end to end delay when compared to DSR routing and 2 hop routing protocol.

\section{References}

[1]. Wu, J., \& Li, H. (2000) Domination and its applications in ad hoc wireless networks with unidirectional links. In Parallel Processing, 2000, International Conference on IEEE, pp. 189-197.

[2]. Breed, G. (2007) Wireless ad hoc networks: Basic concepts. High frequency electronics, pp.44-46.

[3]. Gummalla, A.C.V., \& Limb, J.O. (2000) Wireless medium access control protocols. Communications Surveys \& Tutorials, IEEE, 3(2), pp.2-15.

[4]. Kumar, S., Raghavan, V. S., \& Deng, J. (2006) Medium Access Control protocols for ad hoc wireless networks: A survey. Ad Hoc Networks, 4(3), pp.326-358.

[5]. Langendoen, K., \& Halkes, G. (2005) Energy-efficient medium access control. Embedded Systems Handbook, 34-1.

[6]. Abbas, M. M., \& Mahmood, H. Power Control in Ad Hoc Networks.

[7]. ElBatt, T. A., Krishnamurthy, S. V., Connors, D., \& Dao, S. (2000) Power management for throughput enhancement in wireless adhoc networks. In Communications, 2000. ICC 2000. 2000 IEEE International Conference on IEEE, Vol. 3, pp.1506-1513.

[8]. Almotairi, K. H., \& Shen, X. S. (2012) Distributed power control over multiple channels for ad hoc wireless networks. Wireless Communications and Mobile Computing.

[9]. Kawadia, V., \& Kumar, P. R. (2005) Principles and protocols for power control in wireless ad hoc networks. Selected Areas in Communications, IEEE Journal, 23(1), pp.76-88.

[10]. Belghith, A., \& Akkari, W. (2008, July) Power saving mechanisms for ad hoc networks based on handshaking information tapping. In Proceedings of the Second international conference on Verification and Evaluation of Computer and Communication Systems. British Computer Society. pp. 50-60

[11]. Hiremath, P. S., \& Joshi, S. M. (2012) Energy Efficient Routing Protocol with Adaptive Fuzzy Threshold Energy for MANETs. International Journal of Computer Networks and Wireless Communications (IJCNWC), ISSN: $2250-3501$ Vol, 2.

[12]. Banerjee, A., \& Dutta, P. (2010) Fuzzy-controlled Power-aware Routing Protocol (FPRP) for Mobile Ad Hoc Networks. International Journal of Computer Applications, 11(7), pp.39-43.

[13]. Yu, Z., Fu, X., Cai, Y., \& Vuran, M. C. (2011) A reliable energy-efficient multi-level routing algorithm for wireless sensor networks using fuzzy Petri nets, Sensors, 11(3), pp.3381-3400.

[14]. Lu, D., Huang, X., Liu, C., \& Fan, J. (2010, April) Adaptive power control based spectrum handover for cognitive radio networks. In Wireless Communications and Networking Conference (WCNC), 2010 IEEE, pp. 1-5.

[15]. Zuo, J., Ng, S. X., \& Hanzo, L. (2010, September) Fuzzy logic aided dynamic source routing in cross-layer operation assisted ad hoc networks. In Vehicular Technology Conference Fall (VTC 2010-Fall), 2010 IEEE 72, pp. 1-5.

[16]. Casillas, J., Cordón, O., \& Herrera, F. (2000) Learning fuzzy rules using ant colony optimization algorithms. In Proc. $2^{\text {nd }}$ International Workshop on Ant Algorithms, pp.13-21.

[17]. Agarwal, S., \& Hitzler, P. (2005, November) Modeling fuzzy rules with description logics. In Proceedings of Workshop on OWL Experiences and Directions. Galway, Ireland. 